\title{
RELATIVELY BOUNDED AND COMPACT PERTURBATIONS OF NTH ORDER DIFFERENTIAL OPERATORS
}

\author{
TERRY G. ANDERSON \\ Department of Mathematical Sciences \\ Appalachian State University \\ Boone, NC 28608, U.S.A. \\ E-mail address: tga @math.appstate.edu
}

(Received June 10, 1996 and in revised form August 26, 1996)

\begin{abstract}
A perturbation theory for $n$th order differential operators is developed. For certain classes of operators $L$, necessary and sufficient conditions are obtained for a perturbing operator $B$ to be relatively bounded or relatively compact with respect to $L$. These perturbation conditions involve explicit integral averages of the coefficients of $B$. The proofs involve interpolation inequalities.
\end{abstract}

KEY WORDS AND PHRASES. Perturbation theory, differential operators, relatively bounded, relatively compact, integral averages, interpolation inequalities, maximal and minimal operators, essential spectrum, Fredholm index.

1991 AMS SUBJECT CLASSIFICATION CODES. 34L99, 47E05.

\section{INTRODUCTION AND MAIN RESULTS}

We develop a perturbation theory for $n$th order differential operators. In the following, the differential operator $B$ will be regarded as a perturbation of a (typically) higher-order differential operator $L$. For certain classes of operators $L$, we obtain necessary and sufficient conditions for $B$ to be $L$-bounded or $L$-compact. We employ the following terminology as given in Kato [5, pp. 190, 194].

DEFINITION A. $B$ is relatively bounded with respect to $L$ or simply $L$-bounded if $D(L) \subseteq D(B)$ and $B$ is bounded on $D(L)$ with respect to the graph norm $\|\cdot\|_{L}$ of $L$ defined by $\|y\|_{L}=\|y\|+\|L y\|$, $y \in D(L)$, where $D(L)$ denotes the domain of $L$. In other words, $B$ is $L$-bounded if $D(L) \subseteq D(B)$ and there exist nonnegative constants $\alpha$ and $\beta$ such that

$$
\|B y\| \leq \alpha\|y\|+\beta\|L y\|, \quad y \in D(L) .
$$

The greatest lower bound $\beta_{0}$ of all positive constants $\beta$ for which this inequality holds is called the relative bound of $B$ with respect to $L$ or simply the $L$-bound of $B$. In general, the constant $\alpha$ will increase without bound as $\beta$ is chosen closer to $\beta_{0}$ (so that the infimum $\beta_{0}$ need not be attained). A sequence $\left\{y_{n}\right\}$ is said to be $L$-bounded if there exists $K>0$ such that $\left\|y_{n}\right\|_{L}<K, n \geq 1$.

$B$ is called relatively compact with respect to $L$ or simply $L$-compact if $D(L) \subseteq D(B)$ and $B$ is compact on $D(L)$ with respect to the $L$-norm, i.e., $B$ takes every $L$-bounded sequence into a 
sequence which has a convergent subsequence. For example, if $L$ is the identity map, then $L$ boundedness ( $L$-compactness) of $B$ is equivalent to the usual operator norm boundedness (compactness) of $B$.

The function space setting is the weighted Banach space $L_{w}^{p}(I)$, where $1 \leq p<\infty, W$ is a positive Lebesgue measurable function defined on an interval $I$ of the real line, and $L_{w}^{p}(I)$ denotes the Lebesgue space of equivalence classes of complex-valued functions $y$ with domain $I$ such that $\|y\|:=\left[\int_{1} W|y|^{p}\right]^{1 / p}<\infty$. If $W \equiv 1$, we denote this space by $L^{p}(I)$. The space of complexvalued functions $y$ with domain $I$ such that $\|y\|_{\infty}:=\operatorname{ess}_{t \in I}|y(t)|<\infty$ is denoted by $L^{-}(I)$. A local property is indicated by use of the subscript "loc," and AC is used to abbreviate absolutely continuous. The space of all complex-valued, $n$ times continuously differentiable functions on $I$ is denoted by $C^{n}(I) ; \quad C_{c}^{n}(I)$ denotes the restriction of $C^{n}(I)$ to functions with compact support contained in $I$; and $C_{0}^{\infty}(I)$ is the space of all complex-valued functions on $I$ which are infinitely differentiable and have compact support contained in the interior of $I$. We adopt the definitions of maximal and minimal operators given in Goldberg [4, pp. 127-128, 135].

DEFINITION B. Let $l$ be a differential expression of the form $l=\frac{1}{W^{1 / p}} \sum_{i=0}^{n} a_{i}(t) D^{\prime}$ $\left(D=\frac{d}{d t}\right)$, where $W$ is a positive Lebesgue measurable function defined on $I$ and each $a_{t}$ is a complex-valued function on $I$. Then the maximal operator $L$ corresponding to $l$ has domain $D(L)=\left\{y \in L_{w}^{p}(I): y^{(n-1)} \in A C_{l o c}(I), l[y] \in L_{w}^{p}(I)\right\} \quad$ a n d $\quad$ a c t i o n $L[y]=l[y]=\frac{1}{W^{1 / p}} \sum_{i=0}^{n} a_{t}(t) y^{(i)} \quad(y \in D(L))$. If $a_{t} \in C^{\prime}(I)$ for $0 \leq i \leq n$ and $a_{n} \neq 0$ on $I$, then the minimal operator $L_{0}$ corresponding to $l$ is defined to be the minimal closed extension of $L$ restricted to those $y \in D(L)$ which have compact support in the interior of $I$. In the Hilbert space setting of $L^{2}(I)$, most of the smoothness requirements on the coefficients $a_{1}(0 \leq i \leq n)$ are not needed, and the theory is developed in Naimark [7, sect. 17].

We consider perturbations

$$
B=\frac{1}{W^{1 / p}} \sum_{j=0}^{n-1} b, D^{\prime} \quad(a \leq t<\infty)
$$

of the operators

$$
T=\frac{1}{W^{1 / p}} P^{1 / p} D^{n}
$$

and

$$
L=\frac{1}{W^{1 / p}} \sum_{i=0}^{n} a_{i} P_{i}^{1 / p} D^{i}
$$

in the setting of $L_{w}^{p}(a, \infty)$, where $1 \leq p<\infty$ and $W$ is a positive Lebesgue measurable function defined on $(a, \infty)$. Definitions and conditions for $P$ and $P_{\imath}$ are given in the hypotheses of Theorems 1.1 and 1.2 , respectively. We give conditions on certain averages of the perturbation coefficients $b$, $(0 \leq j \leq n-1)$ which are sufficient and, in some cases necessary, for $B$ to be $T$-bounded or $T$ - 
compact. These results rely heavily on Theorems A and B, which are special cases of Theorem 2.1 in Brown and Hinton [3]. These two theorems give sufficient conditions for weighted interpolation inequalities of the form: there exist $\xi \geq 0, \eta>0, K>0$, and $\varepsilon_{0}>0$ such that for all $\varepsilon \in\left(0, \varepsilon_{0}\right)$ and $y$ in a class $D$ of functions,

$$
\int_{a}^{\infty} N\left|y^{(\nu)}\right|^{p} \leq K\left\{\varepsilon^{-\xi} \int_{a}^{\infty} W|y|^{p}+\varepsilon^{n} \int_{a}^{\infty} P\left|y^{(n)}\right|^{p}\right\}
$$

where $0 \leq j \leq n-1$ and $1 \leq p<\infty$.

Theorem 1.1 gives integral average conditions on $b,(0 \leq j \leq n-1)$ which are necessary and sufficient for $B$ to be $T$-bounded or $T$-compact in the case when $1<p<\infty$ and $P$ and $W$ satisfy the conditions in Theorem 5 in Kwong and Zettl [6]. When $W \equiv 1$, these conditions imply that the coefficients of $T$ are bounded above by the corresponding coefficients of an Euler operator. Furthermore, the perturbation conditions for $T$-compactness of $B$ are sufficient for the essential spectrum and Fredholm index to be invariant under perturbations of $T$ by $B$.

By definition (Goldberg [4, pp. 162-163]), the essential spectrum of $T$, written $\sigma_{e}(T)$, is the set of all complex numbers $\lambda$ such that the range $R(\lambda I-T)$ of $\lambda I-T$ is not closed. The essential resolvent of $T$, written $\rho_{e}(T)$, is the complement of this set. By definition (Goldberg [4, p. 102]), the Fredholm index $\kappa(T)$ is given by $\kappa(T)=\alpha(T)-\beta(T)$, where $\alpha(T)$ is the dimension of the null space of $T$ and $\beta(T)$ is the dimension of $L_{w}^{p}(I) \backslash R(T) . \alpha(T)$ is called the kernel index of $T$, and $\beta(T)$ is called the deficiency index of $T$.

In Theorem 1.2, the results in Theorem 1.1 for the single-term operator $T$ are extended to the multi-term operator $L$. An $n$th order perturbation of $L$ is considered in Corollary 1.1. Sufficient conditions are given for invariance of the essential spectrum and Fredholm index of $L$ under such perturbations.

Theorems 1.1 and 1.2 and Corollary 1.1 provide generalizations of results of Balslev and Gamelin [2] as presented in Goldberg [4, pp. 166-175]. Their work deals with bounded coefficient and Euler operators in the unweighted setting of $L^{p}(a, \infty)$ for $1<p<\infty$.

In Theorem 2.1, the sufficiency conditions in Theorem 1.1 are generalized for operators $T$ with arbitrarily large coefficients. Again, these conditions involve integral averages of the perturbation coefficients $b,(0 \leq j \leq n-1)$. Theorem 2.2 gives pointwise conditions on $b$, $(0 \leq j \leq n-1)$ under which the conclusions of Theorem 2.1 hold. The case in which $p=1$ is covered by Theorem 2.2. Also, perturbation conditions which are sufficient for $L$-boundedness or $L$ compactness of $B$ are obtained for the case $p=1$ and the case in which the coefficients of $L$ are arbitrarily large. These theorems rely heavily on investigations by Brown and Hinton [3] on sufficient conditions for interpolation inequalities. Examples of each theorem are presented and contrasted for the situation in which the coefficient in $T$ is an exponential function.

The final theorem, Theorem 3.1, deals exclusively with the case $p=1$. Sufficient, integral average conditions are given for $T$-boundedness of $B$.

\section{INTEGRAL AVERAGE CONDITIONS FOR EULER-LIKE OPERATORS}

In this section we consider operators whose coefficients are powers of a fixed function $s$ times a weight function $w$ and a bounded function. In the simplest case, i.e., $w(t)=s(t) \equiv 1$, Theorem 1.2 gives Theorem VI.8.1 of [4]. For $\alpha=0, w(t) \equiv 1$, and $s(t)=t$, the sufficiency condition of part (ii) of Theorem 1.2 yields Corollary VI.8.4 of [4] for perturbations of the Euler operator. Since we 
do not require $w(t) \equiv 1$ or $\alpha=0$, we refer to the unperturbed operator of Theorem 1.2 as Eulerlike.

THEOREM 1.1. Let $1<p<\infty$ and $I=[a, \infty)$. Let $s$ and $w$ be positive, $\operatorname{AC}_{\mathrm{loc}}(I)$ functions such that $\left|s^{\prime}(t)\right| \leq N_{0}$ and $\left|s(t) w^{\prime}(t)\right| \leq M_{0} w(t)$ a.e. on I for some constants $N_{0}$ and $M_{0}$. Let $\alpha \in \mathbb{R}$, $W=w s^{\alpha p}$, and $P=w s^{(\alpha+n) p}$. Let $T, B: L_{w}^{p}(a, \infty) \rightarrow L_{w}^{p}(a, \infty)$ be the maximal operators corresponding to the differential expressions $\quad \tau=\frac{1}{W^{1 / p}} P^{1 / p} D^{n} \quad\left(D=\frac{d}{d t}\right) \quad$ and $v=\frac{1}{W^{1 / p}} \sum_{j=0}^{n-1} b_{j} D^{\prime}$, respectively, where each $b_{j} \in L_{\text {oc }}(I)$. For $0 \leq j \leq n-1$ and $\delta>0$, define

$$
g_{\text {J. } \delta}(t)=\frac{1}{s(t)} \int_{t}^{t+\delta s(t)} \frac{\left|b_{,}(\tau)\right|^{p}}{w(\tau) s(\tau)^{(\alpha+j) p}} d \tau
$$

Then the following hold:

(i) $B$ is $T$-bounded if and only if $b, \in L_{\mathrm{loc}}^{p}(I)$ and

$$
\sup _{a \leq 1 \leq-\infty} g_{J, \delta}(t)<\infty \quad(0 \leq j \leq n-1)
$$

for some $\delta \in\left(0,1 /\left(2 N_{0}\right)\right)$. When (1.1) holds, the relative bound for $B$ is 0 . Furthermore, the maximal operator corresponding to $\tau+v$ is $T_{\tau+v}=T+B$.

(ii) $B$ is $T$-compact if and only if $b, \in L_{\text {oc }}^{p}(I)$ and

$$
\lim _{t \rightarrow \infty} g_{J . \delta}(t)=0 \quad(0 \leq j \leq n-1)
$$

for some $\delta \in\left(0,1 /\left(2 N_{0}\right)\right)$. When (1.2) holds, $T$ and $T_{\tau+v}$ have the same essential spectrum and $\lambda \in \rho_{e}(T) \Rightarrow \kappa(\lambda I-T)=\kappa\left(\lambda I-T_{\tau+v}\right)$, where $\rho_{e}(T)$ is the essential resolvent of $T$ and $\kappa(T)$ is the Fredholm index of $T$.

The following theorem is part of Theorem 2.1 in Brown and Hinton [3]. It gives sufficient conditions for weighted interpolation inequalities.

THEOREM A. Let $1 \leq p<\infty, I=[a, \infty)$, and $0 \leq j \leq n-1$. Let $N, W$, and $P$ be positive

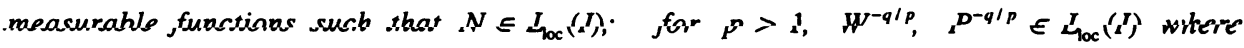
$\frac{1}{p}+\frac{1}{q}=1 ;$ for $p=1, W^{-1}, P^{-1}$ are locally essentially bounded on $I$. Suppose there exists $\varepsilon_{0}>0$ and a positive continuous function $f=f(t)$ on I such that

$$
S_{1}(\varepsilon):=\sup _{t \in I}\left\{f^{(n-\jmath) p} T_{t, \varepsilon}(P)\left[\frac{1}{\varepsilon f} \int_{t}^{t+\varepsilon f} N\right]\right\}<\infty
$$

and

$$
S_{2}(\varepsilon):=\sup _{t \in I}\left\{f^{-J p} T_{t, \varepsilon}(W)\left[\frac{1}{\varepsilon f} \int_{t}^{t+\varepsilon f} N\right]\right\}<\infty
$$


for all $\varepsilon \in\left(0, \varepsilon_{0}\right)$, where

$$
T_{t, \varepsilon}(P)=\left\{\begin{array}{cl}
\left\|P^{-1}\right\|_{\infty,|t, t+\varepsilon f|}, & p=1 \\
{\left[\frac{1}{\varepsilon f} \int_{t}^{t+\varepsilon f} P^{-q / p}\right]^{p / q},} & 1<p<\infty
\end{array}\right.
$$

with similar definitions for $T_{t, \varepsilon}(W)$. Then there exists $K>0$ such that for all $\varepsilon \in\left(0, \varepsilon_{0}\right)$ and $y \in D$,

$$
\int_{1} N\left|y^{(\jmath)}\right|^{p} \leq K\left\{\varepsilon^{-\jmath p} S_{2}(\varepsilon) \int_{1} W|y|^{p}+\varepsilon^{(n-\jmath) p} S_{1}(\varepsilon) \int_{1} P\left|y^{(n)}\right|^{p}\right\}
$$

where $D=\left\{y: y^{(n-1)} \in \operatorname{AC}_{\mathrm{loc}}(I), \int_{1} W|y|^{p}<\infty\right.$, and $\left.\int_{1} P\left|y^{(n)}\right|^{p}<\infty\right\}$.

\section{PROOF OF THEOREM 1.1.}

(i) Sufficiency. Suppose (1.1) holds for some $\delta \in\left(0,1 /\left(2 N_{0}\right)\right)$. We will show that Theorem A applies to the choices $f=s, N=\left|b_{j}\right|^{p}, \varepsilon_{0}=\delta$, and $W$ and $P$ as in Theorem 1.1. Basic estimates are obtained from the following lemma in [3, pp. 575-576].

LEMMA A. Let $s$ and $w$ be as in Theorem 1.1. Then for fixed $t \in I, \quad 0<\varepsilon<1 / N_{0}$, and $t \leq \tau \leq t+\varepsilon s(t)$, we have that $\quad\left(1-\varepsilon N_{0}\right) s(t) \leq s(\tau) \leq\left(1+\varepsilon N_{0}\right) s(t) \quad$ and $\exp \left(-\frac{M_{0}}{N_{0}}\right) w(t) \leq w(\tau) \leq \exp \left(\frac{M_{0}}{N_{0}}\right) w(t)$.

This implies that both positive and negative powers of $s(\tau)$ and $w(\tau)$ are essentially constant for $t \leq \tau \leq t+\varepsilon s(t)$ and fixed $t$. By Lemma A and the definitions of $P$ and $W$,

$$
T_{t, \varepsilon}(P)=\left[\frac{1}{\varepsilon s(t)} \int_{t}^{t+\varepsilon s(t)} w(\tau)^{-\alpha p} s(\tau)^{-(\alpha+n) q} d \tau\right]^{p / q} \leq C_{1} w(t)^{-1} s(t)^{-(\alpha+n) p}
$$

and similarly

$$
T_{t, \varepsilon}(W) \leq C_{2} w(t)^{-1} s(t)^{-\alpha p}
$$

for all $t \in I$ and $\varepsilon \in(0, \delta)$, where $C_{1}$ and $C_{2}$ are independent of $t$ and $\varepsilon$. Using Lemma $A$ again, we obtain for a constant $C_{3}$,

$$
\begin{gathered}
\frac{1}{\varepsilon f(t)} \int_{t}^{t+\varepsilon f(t)} N=\frac{1}{\varepsilon s(t)} \int_{t}^{t+\varepsilon s(t)}|b|^{p} \leq \frac{C_{3} w(t) s(t)^{(\alpha+\jmath) p}}{\varepsilon} \frac{1}{s(t)} \int_{t}^{t+\varepsilon(t)} \frac{|b|^{p}}{w s^{(\alpha+j) p}} \\
\leq \frac{C_{3}}{\varepsilon} w(t) s(t)^{(\alpha+\jmath) p} g_{ر, \delta}(t)
\end{gathered}
$$

for all $t \in I, \varepsilon \in(0, \delta)$. Hence, by (1.1), there is a constant $C>0$ such that 


$$
\frac{1}{\varepsilon f(t)} \int_{t}^{t+\varepsilon f(t)} N \leq \frac{C}{\varepsilon} w(t) s(t)^{(\alpha+j) p}
$$

for all $t \in I, \varepsilon \in(0, \delta)$. Thus

$$
S_{1}(\varepsilon) \leq \sup _{t \in I}\left\{s(t)^{(n-\jmath) p} C_{1} w(t)^{-1} s(t)^{-(\alpha+n) p} \frac{C}{\varepsilon} w(t) s(t)^{(\alpha+\jmath) p}\right\}
$$

so that

$$
S_{1}(\varepsilon) \leq \frac{C C_{1}}{\varepsilon}, \quad 0<\varepsilon<\delta .
$$

Similarly,

$$
S_{2}(\varepsilon) \leq \frac{C C_{2}}{\varepsilon}, \quad 0<\varepsilon<\delta .
$$

Hence, by Theorem A, there is a constant $K$ such that for all $y \in D=D(T)$,

$$
\int_{I}\left|b, y^{(\jmath)}\right|^{p} \leq K\left\{\varepsilon^{-\jmath p-1} \int_{I} W|y|^{p}+\varepsilon^{(n-\jmath) p-1} \int_{I} P\left|y^{(n)}\right|^{p}\right\} .
$$

Use of the elementary inequality $\left(a^{p}+b^{p}\right)^{1 / p} \leq a+b(a, b \geq 0)$ gives

$$
\left\|\frac{1}{W^{1 / p}} b, y^{(j)}\right\| \leq K_{1} \varepsilon^{(-\jmath-1 / p)}\|y\|+K_{1} \varepsilon^{(n-J-1 / p)}\|T y\|
$$

for all $y \in D(T), 0 \leq j \leq n-1$, where $K_{1}=K^{1 / p}$. Restrict $\varepsilon \leq 1$. Then the right side can be bounded above independently of $j$, and the triangle inequality gives

$$
\|B y\| \leq K_{1} \varepsilon^{(-n+1-1 / p)}\|y\|+K_{1} \varepsilon^{(1-1 / p)}\|T y\|
$$

for all $y \in D(T)$. Since $p>1$, it follows that $B$ is $T$-bounded with relative bound 0 . The result $T_{\tau+\nu}=T+B$ follows by an argument given on pp. 169-170 in Goldberg [4].

Necessity. Suppose $B$ is $T$-bounded. Let $\phi$ be a function in $C_{0}^{\infty}(\mathbf{R})$ such that $\phi \equiv 1$ on $[0,1]$ and $\operatorname{support}(\phi)=[-2,2]$. Fix $\delta \in\left(0,1 /\left(2 N_{0}\right)\right)$. For each $r \geq a$, define

$$
\phi_{r}(t)=\phi\left(\frac{t-r}{\delta s(r)}\right), \quad t \geq a .
$$

Then $\phi_{r} \equiv 1$ on $[r, r+\delta s(r)]$ and $\operatorname{support}\left(\phi_{r}\right)=[r-2 \delta s(r), r+2 \delta s(r)]$. We proceed by an induction argument. First consider $j=0$ in (1.1). Fix $r \geq a$. Note that $B \phi_{r}=\frac{1}{W^{1 / p}} b_{0}$ on 
$[r, r+\delta s(r)]$, so that $g_{0 . \delta}(r)=\frac{1}{s(r)} \int_{r}^{r+\delta s(r)} \frac{W\left|B \phi_{r}\right|^{p}}{w s^{\alpha p}}$. Now, applying Lemma A, there is a constant $C$ independent of $r$ such that

$$
\begin{aligned}
g_{0, \delta}(r) & \leq \frac{C}{w(r) s(r)^{1+\alpha_{p}}} \int_{r}^{r+\delta s(r)} W\left|B \phi_{r}\right|^{p} \leq \frac{C}{w(r) s(r)^{1+\alpha_{p}}}\left\|B \phi_{r}\right\|^{p} \\
& \leq \frac{K}{w(r) s(r)^{1+\alpha_{p}}}\left(\left\|\phi_{r}\right\|^{p}+\left\|T \phi_{r}\right\|^{p}\right)
\end{aligned}
$$

for some constant $K$ independent of $r$, where the last inequality follows from the hypothesis that $B$ is $T$-bounded.

Using the compact support of $\phi_{r}$, Lemma $A$, a change of variable, and the fact that $\phi \in C_{0}^{\infty}(\mathbb{R})$, we have for some constant $C_{0}$,

$$
\begin{aligned}
\left\|\phi_{r}\right\|^{p} & =\int_{r-2 \delta s(r)}^{r+2 \delta s(r)} w s^{\alpha_{p}}\left|\phi_{r}\right|^{p} \leq C w(r) s(r)^{\alpha_{p}} \int_{r-2 \delta s(r)}^{r+2 \delta s(r)}\left|\phi\left(\frac{t-r}{\delta s(r)}\right)\right|^{p} d t \\
& \leq C w(r) s(r)^{\alpha_{p}} \int_{-\infty}^{\infty}|\phi(u)|^{p} \delta s(r) d u \\
& \leq C_{1} w(r) s(r)^{\alpha_{p+1}}
\end{aligned}
$$

for some constant $C_{1}$ independent of $r$. Similarly, for some $C_{0}$,

$$
\begin{aligned}
\left\|T \phi_{r}\right\|^{p} & =\int_{a}^{\infty} W\left|T \phi_{r}\right|^{p}=\int_{a}^{\infty} P\left|\phi_{r}^{(n)}\right|^{p}=\int_{r-2 \delta s(r)}^{r+2 \delta s(r)} w s^{(\alpha+n) p}\left|\phi_{r}^{(n)}\right|^{p} \\
& \leq C_{0} w(r) s(r)^{(\alpha+n) p} \int_{-\infty}^{\infty} \mid \frac{d^{n}}{d t^{n}} \phi\left(\left.\frac{t-r}{\delta s(r)}\right|^{p} d t\right. \\
& =C_{0} w(r) s(r)^{(\alpha+n) p} \int_{-\infty}^{\infty}\left|\phi^{(n)}(u) \frac{1}{\delta^{n} s(r)^{n}}\right|^{p} \delta s(r) d t \\
& \leq C_{2} w(r) s(r)^{\alpha p+1}
\end{aligned}
$$

for some constant $C_{2}$ independent of $r$. Use of (1.11) and (1.12) in (1.10) yields $g_{0 . \delta}(r) \leq K\left(C_{1}+C_{2}\right), \quad r \in[a, \infty)$. Therefore, (1.1) holds for $j=0$ and all $\delta \in\left(0,1 /\left(2 N_{0}\right)\right)$.

Next fix $k \leq n-1$. Suppose (1.1) holds for $0 \leq j \leq k-1$ and some $\delta \in\left(0,1 /\left(2 N_{0}\right)\right)$. Let $A$ be the maximal operator with action given by $A=\frac{-1}{W^{1 / p}} \sum_{j=0}^{k-1} b_{j} D^{\prime} . \quad$ By the sufficiency argument above, $A$ is $T$-bounded. Thus since $B$ is $T$-bounded, Minkowski's inequality implies that $A$ $+B$ is $T$-bounded. Note that $(A+B) y=\frac{1}{W^{1 / p}} \sum_{j=k}^{n-1} b, y^{(j)}, \quad y \in D(T)$. With $\phi$ and $\phi_{r}$ defined as above (see (1.9)), define 


$$
h(t)=\phi(t) \frac{t^{k}}{k !}, \quad t \geq a
$$

Then $h \in C_{0}^{\infty}(\mathbb{R})$ and $h^{(k)} \equiv 1$ on $[0,1]$. For each $r \geq a$, define

$$
h_{r}(t)=\delta^{k} s(r)^{k} h(u), \quad t \geq a,
$$

where $\quad u=\frac{t-r}{\delta s(r)}$. Then $\quad h_{r}^{(k)}(t)=h^{(k)}(u), \quad h_{r}^{(k)}(t)=1 \quad$ for $\quad r \leq t \leq r+\delta s(r), \quad$ and $\operatorname{support}\left(h_{r}\right)=[r-2 \delta s(r), r+2 \delta s(r)]$. Thus

$$
(A+B) h_{r}=\frac{b_{k}}{W^{1 / p}} \quad \text { on }[r, r+\delta s(r)]
$$

By Lemma A, we obtain for a constant $C$,

$$
\begin{aligned}
g_{k, \delta}(r) & =\frac{1}{s(r)} \int_{r}^{r+\delta s(r)} \frac{\left|b_{k}\right|^{p}}{w s^{(\alpha+k) p}}=\frac{1}{s(r)} \int_{r}^{r+\delta s(r)} \frac{W\left|(A+B) h_{r}\right|^{p}}{w s^{(\alpha+k) p}} \\
& \leq \frac{C}{w(r) s(r)^{(\alpha+k) p+1}} \int_{r}^{r+\delta s(r)} W\left|(A+B) h_{r}\right|^{p} \leq \frac{C}{w(r) s(r)^{(\alpha+k) p+1}}\left\|(A+B) h_{r}\right\|^{p} \\
& \leq \frac{C}{w(r) s(r)^{(\alpha+k) p+1}}\left(\left\|h_{r}\right\|^{p}+\left\|T h_{r}\right\|^{p}\right),
\end{aligned}
$$

where the last inequality follows from the relative boundedness of $A+B$ with respect to $T$. By calculations like those used in deriving (1.11) and (1.12), we obtain for $r \geq a$,

$$
\left\|h_{r}\right\|^{p} \leq C_{1} w(r) s(r)^{(\alpha+k) p+1}
$$

and

$$
\left\|T h_{r}\right\|^{p} \leq C_{2} w(r) s(r)^{(\alpha+k) p+1}
$$

where $C_{1}$ and $C_{2}$ are constants independent of $r$. Thus (1.6) implies that (1.1) holds for $j=k$ and any $\delta \in\left(0,1 /\left(2 N_{0}\right)\right)$. This establishes necessity of (1.1).

(ii) Sufficiency. Suppose (1.2) holds for some $\delta \in\left(0,1 /\left(2 N_{0}\right)\right)$. We will use an argument similar to that in Goldberg [4, pp. 171-172]. For each positive integer $N>a$, define $B_{N}$ on $D(T)$ by $\quad B_{N} y=\left\{\begin{array}{ll}B y & \text { on }[a, N], \\ 0 & \text { on }(N, \infty) .\end{array}\right.$ We show that $B_{N}$ converges to $B$ in the space of bounded operators on $D(T)$ with the $T$-norm. First note that $T$ is closed. To see this, let $f_{n} \rightarrow f$ and $T f_{n} \rightarrow g$ in $L_{w}^{p}(a, \infty)$. Let $\mathrm{J}$ be a compact subinterval of $[a, \infty)$ and restrict the functions $f$, $f_{n}$, and $g$ to J. Define $T_{\mathrm{J}}: L_{w}^{p}(\mathrm{~J}) \rightarrow L_{w}^{p}(\mathrm{~J})$ to be the maximal operator corresponding to $\tau$ on $\mathrm{J}$. Clearly, $f_{n} \rightarrow f$ in $L_{w}^{p}(\mathrm{~J})$ and $f_{n} \in D\left(T_{\mathrm{j}}\right)$. Since $T_{\mathrm{j}} f_{n}=\left(T f_{n}\right)_{j}, T_{\mathrm{j}} f_{n} \rightarrow g$ in $L_{w}^{p}(\mathrm{~J})$. By Theorems VI.3.1 and IV.1.7 in Goldberg [4], $T_{\mathrm{j}}$ is closed. Therefore, $f \in D\left(T_{\mathrm{j}}\right)$ and $T_{\mathrm{j}} f=g$. Thus, $f \in D(T)$ and $T f=g$. Hence $T$ is closed. 
Therefore $D(T)$ is complete under the $T$-norm. From (i), $B$ is $T$-bounded. So $D(T) \subseteq D(B)$. For $y \in D(T)$,

$$
\left\|B y-B_{N} y\right\|=\left\{\int_{a}^{\infty} W\left|B y-B_{N} y\right|^{p}\right\}^{1 / p}=\left\{\int_{N}^{\infty} W|B y|^{p}\right\}^{1 / p} \leq \sum_{j=0}^{n-1} \int_{N}^{\infty}\left|b_{J} y^{(\jmath)}\right|^{p}
$$

By the argument used in proving sufficiency in (i), Theorem A applies to the interval $I=[N, \infty)$ with the same choices for the weights, $f$, and $\varepsilon_{0}$. By (1.3) and (1.4), for $0<\varepsilon<\delta$,

$$
S_{1}(\varepsilon) \leq C_{1} \sup _{t \in[N, \infty)}\left\{w(t)^{-1} s(t)^{-(\alpha+j) p} \frac{1}{\varepsilon s(t)} \int_{t}^{t+\varepsilon s(t)}\left|b_{J}\right|^{p}\right\}
$$

and the same estimate holds for $S_{2}(\varepsilon)$ up to a multiplicative constant. By Lemma A, for $0<\varepsilon<\delta$,

$$
\frac{1}{\varepsilon s(t)} \int_{t}^{t+\varepsilon s(t)}|b|^{p} \leq \frac{C}{\varepsilon} w(t) s(t)^{(\alpha+\jmath) p} g_{, \delta}(t), \quad t \in[N, \infty) .
$$

Hence

$$
S_{1}(\varepsilon) \leq \frac{C}{\varepsilon} \sup _{t \in \mid N, \infty)} g_{J, \delta}(t)
$$

with a similar estimate for $S_{2}(\varepsilon), 0<\varepsilon<\delta$, where $C$ is a constant independent of $N$ and $\varepsilon$. It follows from Theorem A that for all $y \in D(T)$,

$$
\begin{aligned}
\int_{N}^{\infty}\left|b, y^{(J)}\right|^{p} \leq & \frac{K}{\varepsilon}\left\{\varepsilon^{-J p} \int_{a}^{\infty} W|y|^{p}+\varepsilon^{(n-\jmath) p} \int_{a}^{\infty} P\left|y^{(n)}\right|^{p}\right\}\left[\sup _{I \in[N, \infty)} g_{J, \delta}(t)\right] \\
& \leq C_{J}\|y\|_{T}\left[\sup _{t \in[N, \infty)} g_{J, \delta}(t)\right],
\end{aligned}
$$

where $C$, is independent of $y$ and $N$ (but depends on $\varepsilon$ ). Use of (1.23) in (1.19) gives

$$
\frac{\left\|B y-B_{N} y\right\|}{\|y\|_{T}} \leq \sum_{j=0}^{n-1} C_{j}\left[\sup _{t \in(N,-)} g_{J, \delta}(t)\right]
$$

for all $y \in D(T)$ such that $y \neq 0$. By (1.2), the term on the right side approaches 0 as $N \rightarrow \infty$. Therefore, $B_{N} \rightarrow B$ in the space of bounded operators on $D(T)$ with the $T$-norm.

Next, we show that each $B_{N}$ is $T$-compact. Let $\left\{f_{l}\right\}$ be a $T$-bounded sequence, say $\left\|f_{i}\right\|_{T} \leq \gamma$ for all $l$. We will show that $\left\{f_{l}^{(j)}\right\}, 0 \leq j \leq n-1$, is uniformly bounded on $[a, N]$. Partition $I=[a, N]$ by $J_{t}=\left[t_{t}, t_{t+1}\right], \quad 1 \leq i \leq k$, with $t_{1}=a, \quad t_{t+1}=t_{t}+\varepsilon s\left(t_{t}\right)$, and $\varepsilon \in(0, \delta)$ chosen such that $N=t_{k+1}=t_{k}+\varepsilon s\left(t_{k}\right)$. From the proof of Theorem 2.1 in Brown and Hinton [3], with $t \in J_{\imath}$,

$$
\left|f_{i}^{(j)}(t)\right|^{p} \leq K\left\{\left[\varepsilon \mathrm{s}\left(t_{t}\right)\right]^{-J p} T_{t_{1}, \varepsilon}(W) \frac{1}{\varepsilon \mathrm{s}\left(t_{t}\right)} \int_{J_{1}} W\left|f_{l^{\prime}}\right|^{p}+\left[\varepsilon \mathrm{s}\left(t_{t}\right)\right]^{(n-\jmath) p} T_{t_{1}, \varepsilon}(P) \frac{1}{\varepsilon \mathrm{s}\left(t_{t}\right)} \int_{J_{1}} P\left|f_{l}^{(n)}\right|^{p}\right\}
$$


Use of (1.3) and (1.4) yields for some $C_{0}$ (depending on $\varepsilon$ ),

$$
\left|f_{l}^{(J)}(t)\right|^{p} \leq \frac{C_{0}}{w\left(t_{1}\right) \mathrm{s}\left(t_{1}\right)^{(\alpha+j) p+1}}\left\{\int_{J_{l}} W\left|f_{l}\right|^{p}+\int_{J_{l}} P\left|f_{l}^{(n)}\right|^{p}\right\}
$$

for $t \in J_{1}$. Since $w$ and $s$ are positive, continuous functions on $[a, \infty)$ and $t_{t} \in J_{t} \subset[a, N]$, we have for some $C$ depending on $\varepsilon$,

$$
\left|f^{(\jmath)}(t)\right|^{p} \leq C\left\{\int_{\mathrm{a}}^{\infty} W\left|f_{l}\right|^{p}+\int_{\mathrm{a}}^{\infty} W\left|T f_{l}\right|^{p}\right\}=C\left\|f_{i}\right\|_{T}
$$

for $t \in[a, N], 0 \leq j \leq n-1$. Since $\left\{f_{l}\right\}$ is $T$-bounded, $\left\{f_{l}^{(j)}\right\}, \quad 0 \leq j \leq n-1$, is uniformly bounded on $[a, N]$.

Next we show $\left\{f_{l}^{(j)}\right\}, 0 \leq j \leq n-1$, is equicontinuous on $[a, N]$. Let $\eta>0$ be given. For $t, s \in[a, N]$,

$$
\left|f_{l}^{(j)}(t)-f_{l}^{(j)}(s)\right|=\left|\int_{s}^{t} f_{l}^{(j+1)}\right| \leq\left.\left.\left.\left|\int_{s}^{t} \frac{1}{\mathrm{~W}^{1 / p}} \mathrm{~W}^{1 / p}\right| f_{l}^{(j+1)}|\leq| \int_{s}^{t} \frac{1}{\mathrm{~W}^{\alpha / p}}\right|^{1 / q}\left|\int_{s}^{t} \mathrm{~W}\right| f_{l}^{(j+1)}\right|^{p}\right|^{1 / p}
$$

by Holder's inequality, where $\frac{1}{p}+\frac{1}{q}=1$. Since $w$ and $s$ are positive, continuous functions on $[a, \infty), W=w s^{\alpha p}$ is bounded above and below on $[a, N]$. Hence for $t, s \in[a, N]$,

$$
\left|f_{l}^{(j)}(t)-f_{l}^{(j)}(s)\right| \leq C|t-s|^{1 / 9}\left\|f_{l}^{(j+1)}\right\|_{L_{w}^{p}(a, N)}
$$

where the constant $C$ depends on $W$. For the case $0 \leq j \leq n-2$, the argument used to obtain (1.25) applies to $j+1 \leq n-1$ and yields $\left|f_{i}^{(j+1)}(t)\right| \leq C\left\|f_{i}\right\|_{r}, \quad t \in[a, N]$. This implies that, since $W$ is bounded on $[a, N]$, with a new $C$,

$$
\left\|f_{l}^{(j+1)}\right\|_{L_{w}^{p}(a, N)} \leq C\left\|f_{l}\right\|_{T}, \quad 0 \leq j \leq n-2 .
$$

For the case $j=n-1$,

$$
\left\|f_{l}^{(j+1)}\right\|_{L_{w}^{p}(a, N)}=\left\|f_{l}^{(n)}\right\|_{L_{w}^{p}(a, N)} \leq\left\{\int_{a}^{N} W \mid T f_{l}^{p}\right\}^{1 / p} \leq C\left\|T f_{l}\right\| \leq C\left\|f_{l}\right\|_{T}
$$

since $W / P$ is bounded on $[a, N]$. Thus, in any case, (1.28) holds for $0 \leq j \leq n-1$. So (1.26) implies that

$$
\left|f_{l}^{(j)}(t)-f_{l}^{(j)}(s)\right| \leq C|t-s|^{1 / q}\left\|f_{i}\right\|_{T} \leq M|t-s|^{1 / q}
$$

where $\quad M=C \sup \left\{\left\|f_{l}\right\|_{T}: l \geq 1\right\}$, since $\left\{f_{l}\right\}$ is $T$-bounded. Since $p>1, \quad 1 / q>0$. Therefore, $\left\{f_{l}^{(j)}\right\}$ is equicontinuous and bounded on $[a, N], 0 \leq j \leq n-1$. By the Arzela-Ascoli Theorem, $\left\{f_{i}\right\}$ has a subsequence $\left\{f_{i, 0}\right\}$ which converges uniformly on $[a, N]$, and $\left\{f_{i, 0}^{\prime}\right\}$ has a subsequence $\left\{f_{l, 1}^{\prime}\right\}$ which converges uniformly on $[a, N]$. Hence $\left\{f_{l, 1}\right\}$ and $\left\{f_{l, 1}^{\prime}\right\}$ converge 
uniformly on $[a, N]$. Repeating this procedure, a subsequence $\left\{g_{l}\right\}$ of $\left\{f_{l}\right\}$ is obtained such that for $0 \leq j \leq n-1,\left\{g_{l}^{(j)}\right\}$ converges uniformly on $[a, N]$. By definition of $B_{N}$,

$$
\begin{aligned}
\| B_{N} g_{l} & -B_{N} g_{m} \|=\left\{\int_{a}^{N} W\left|B g_{l}-\mathrm{B} g_{m}\right|^{p}\right\}^{1 / p} \\
& \leq \sum_{j=0}^{\mathrm{n}-1}\left[\sup _{l \in[a, N]}\left|g_{l}^{(j)}(t)-g_{m}^{(j)}(t)\right|\right]\left\{\int_{a}^{N}|b J|\right\}^{1 / p} .
\end{aligned}
$$

It follows that $\left\{B_{N} g_{l}\right\}$ converges in $L_{w}^{p}(a, \infty)$ as $l \rightarrow \infty$. Thus $B_{N}$ is $T$-compact for each $N$, and so $B$ is $T$-compact, being the uniform limit of $T$-compact operators.

Necessity. Suppose $B$ is $T$-compact. First we show that (1.2) holds for $j=0$. We proceed by a contradiction argument. Suppose that for any $\delta \in\left(0,1 /\left(2 N_{0}\right)\right)$, there exists $\varepsilon>0$ and a sequence $\left\{r_{l}\right\}_{l=1}^{\infty}$ of positive numbers such that $r_{l} \rightarrow \infty$ and

$$
\frac{1}{s\left(r_{l}\right)} \int_{r_{l}}^{r_{1}+\delta s\left(r_{l}\right)} \frac{\left|b_{0}\right|^{p}}{w s^{\alpha p}} \geq \varepsilon, \quad l \geq 1 .
$$

Fix $\delta \in\left(0,1 /\left(2 N_{0}\right)\right)$. Let $\left\{\phi_{r}\right\}$ be the functions defined by (1.9). As before,

$$
B \phi_{r}=\frac{1}{W^{1 / p}} b_{0}, \quad \text { on }[r, r+\delta s(r)]
$$

It follows from (1.31) and Lemma $A$ that

$$
\begin{gathered}
\varepsilon \leq \frac{1}{s\left(r_{l}\right)} \int_{r_{l}}^{r_{1}+\delta s\left(r_{l}\right)} \frac{1}{w s^{\alpha p}} W\left|B \phi_{r_{1}}\right|^{p} \leq \frac{C_{0}}{w\left(r_{l}\right) s\left(r_{l}\right)^{1+\alpha_{p}}} \int_{a}^{\infty} W\left|B \phi_{r_{l}}\right|^{p} \\
=\frac{C_{0}}{w\left(r_{l}\right) s\left(r_{l}\right)^{1+\alpha p}}\left\|B \phi_{r_{l}}\right\|^{p}
\end{gathered}
$$

where $C_{0}$ is a constant independent of $l$. For each $r \geq a$, define

$$
\psi_{r}(t)=\frac{1}{w(r)^{1 / p} s(r)^{\alpha+1 / p}} \phi_{r}(t), \quad t \in[a, \infty) .
$$

Then

$$
\left\|\psi_{r_{1}}\right\|_{T}^{p}=\frac{1}{w\left(r_{l}\right) s\left(r_{l}\right)^{1+\alpha p}}\left\|\phi_{r_{1}}\right\|_{T}^{p}
$$

and (1.33) implies that

$$
\varepsilon \leq C_{0}\left\|B \psi_{r_{1}}\right\|^{p}
$$


By (1.11), (1.12), and (1.35), $\left\{\psi_{r_{1}}\right\}$ is $T$-bounded. Since $B$ is $T$-compact, $\left\{B \psi_{r_{1}}\right\}$ has a convergent subsequence. Relabel indices so that $\left\{B \psi_{r_{1}}\right\}$ converges in $L_{w}^{p}(a, \infty)$ to some $y_{0}$. We show that $y_{0}=0$ a.e. in $[a, \infty)$. Let $\mathrm{J}_{0}$ be a finite subinterval of $[a, \infty)$. Since $r_{l} \rightarrow \infty$ as $l \rightarrow \infty$ and $\operatorname{support}\left(\psi_{r_{1}}\right)=\left[r_{t}-2 \delta s\left(r_{l}\right), r_{l}+2 \delta s\left(r_{l}\right)\right]$, we have $\psi_{r_{1}} \equiv 0$ on $\mathrm{J}_{0}$ and $B \psi_{r_{1}} \equiv 0$ on $\mathrm{J}_{0}$ for $l$ sufficiently large. For such $l, \quad\left\|y_{0}\right\|_{L_{w}^{p}\left(J_{0}\right)}=\left\|y_{0}-B \psi_{r_{1}}\right\|_{L_{w}^{p}\left(J_{0}\right)} \leq\left\|y_{0}-B \psi_{r_{1}}\right\|$. Since $B \psi_{r_{i}} \rightarrow y_{0}$ as $l \rightarrow \infty$ and the term on the left side is independent of $l,\left\|y_{0}\right\|_{L_{w}\left(J_{0}\right)}=0$. This holds for an arbitrary finite subinterval $\mathrm{J}_{0}$ of $[a, \infty)$, and so $y_{0}=0$ a.e. in $[a, \infty)$. Therefore, $B \psi_{r_{1}} \rightarrow 0$ in $L_{w}^{p}(a, \infty)$ as $l \rightarrow \infty$. This contradicts (1.36). Thus (1.2) holds for $j=0$.

To establish (1.2) for $1 \leq j \leq n-1$, we use an induction argument. Fix $k \leq n-1$. Suppose (1.2) holds for $0 \leq j \leq k-1$ and some $\delta \in\left(0,1 /\left(2 N_{0}\right)\right)$. Suppose (1.2) does not hold for $j=k$. Then there exists $\varepsilon_{0}>0$ and a sequence $\left\{r_{l}\right\}$ of positive numbers such that $r_{l} \rightarrow \infty$ as $l \rightarrow \infty$ and

$$
g_{k, \delta}\left(r_{l}\right) \geq \varepsilon_{0}, \quad l \geq 1
$$

As in the proof of necessity in (i), let $A$ be the maximal operator with action defined by $A=\frac{-1}{W^{1 / p}} \sum_{j=0}^{k-1} b, D^{\prime} . \quad$ Then $A$ is $T$-compact by the sufficiency argument in (ii). Since $B$ is $T$ compact, $B$ is $T$-bounded. Therefore, the estimate preceding (1.16) yields, with $h_{r}$ as in (1.14),

$$
g_{k . \delta}\left(r_{l}\right) \leq \frac{C}{w\left(r_{l}\right) s\left(r_{l}\right)^{(\alpha+k) p+1}}\left\|(A+B) h_{r_{1}}\right\|^{p}
$$

For each $r \geq a$, define

$$
p_{r}(t)=\frac{1}{w(r)^{1 / p} s(r)^{\alpha+k+1 / p}} h_{r}(t), \quad t \geq a
$$

Then

$$
g_{k, \delta}\left(r_{l}\right) \leq C\left\|(A+B) p_{r_{l}}\right\|^{p}
$$

and

$$
\left\|p_{r_{l}}\right\|_{T}=\frac{1}{w\left(r_{l}\right)^{1 / p} s\left(r_{l}\right)^{\alpha+k+1 / p}}\left(\left\|h_{r_{l}}\right\|+\left\|T h_{r_{l}}\right\|\right) .
$$

By (1.17) and (1.18), $\left\{p_{r_{1}}\right\}$ is $T$-bounded. Since $A$ and $B$ are both $T$-compact, $A+B$ is $T$-compact. Therefore, $\left\{(A+B) p_{r_{1}}\right\}$ contains a convergent subsequence, say (after relabeling indices) $(A+B) p_{r_{1}} \rightarrow z_{0}$ in $L_{w}^{p}(a, \infty)$. We show that $z_{0}=0$ a.e. on $[a, \infty)$. Let $\mathrm{J}_{0} \subset[a, \infty)$ be a finite interval. Since support $\left(p_{r}\right)=[r-2 \delta s(r), r+2 \delta s(r)], \quad p_{r_{i}} \equiv 0 \quad$ on $\mathrm{J}_{0}$ and hence $(A+B) p_{r_{1}} \equiv 0$ on $\mathrm{J}_{0}$ for all $l$ sufficiently large. For such $l$,

$$
\left\|z_{0}\right\|_{L_{w}^{p}\left(\mathrm{~J}_{0}\right)}^{p}=\int_{\mathrm{J}_{0}} W\left|z_{0}(t)-(A+B) p_{r_{i}}(t)\right|^{p} d t \leq\left\|z_{0}-(A+B) p_{r_{r}}\right\|^{p} \rightarrow 0 \quad(l \rightarrow \infty) .
$$


Thus $\int_{\mathrm{J}_{0}} W\left|z_{0}\right|^{p}=0$ for any finite subinterval $\mathrm{J}_{0}$ of $[a, \infty)$. Therefore, $z_{0}=0$ a.e. on $[a, \infty)$ and $(A+B) p_{r_{i}} \rightarrow 0$ in $L_{w}^{p}(a, \infty)$. Hence (1.40) implies that $g_{k, \delta}\left(r_{l}\right) \rightarrow 0$ as $l \rightarrow \infty$, contradicting (1.37). Therefore, (1.2) holds for $i=k$. This establishes necessity of (1.2) for $T$ compactness of $B$. Thus Theorem 1.1 is proved.

Note that Theorem 1.1 deals with perturbations of a single-term operator $T$. In the next theorem, we extend Theorem 1.1 to a multi-term operator $L$.

THEOREM 1.2. Let $p, s, w, W, P, B$, and $g_{, \delta}$ be as in Theorem 1.1. Let $L: L_{w}^{p}(a, \infty) \rightarrow L_{w}^{p}(a, \infty)$ be the maximal operator corresponding to

$$
l=\frac{1}{W^{1 / p}} \sum_{i=0}^{n} a_{i} P_{t}^{1 / p} D^{i}
$$

where $\frac{1}{a_{n}}, a_{i}(0 \leq i \leq n) \in L^{\infty}(a, \infty)$ and $P_{1}=w s^{(\alpha+i) p}$. Then the following hold:

(i) $B$ is L-bounded if and only if $b, \in L_{l o c}^{p}(a, \infty)$ and

$$
\sup _{a \leq t<\infty} g_{, \delta}(t)<\infty \quad(0 \leq j \leq n-1)
$$

for some $\delta \in\left(0,1 /\left(2 N_{0}\right)\right)$. When (1.42) holds, the relative bound for $B$ is 0 . Furthermore, the maximal operator corresponding to $l+v$ is $L_{l+v}=L+B$.

(ii) $B$ is L-compact if and only if $b_{j} \in L_{l o c}^{p}(a, \infty)$ and

$$
\lim _{t \rightarrow \infty} g_{J, \delta}(t)=0 \quad(0 \leq j \leq n-1)
$$

for some $\delta \in\left(0,1 /\left(2 N_{0}\right)\right)$. When (1.43) holds, $L$ and $L_{l+v}$ have the same essential spectrum and $\lambda \in \rho_{e}(L) \Rightarrow \kappa(\lambda I-L)=\kappa\left(\lambda I-L_{l+v}\right)$.

To prove Theorem 1.2, we will use the following lemmas.

LEMMA 1.1. Suppose $A, C$, and $D$ are linear operators such that $D$ is $C$-bounded with relative bound less than 1 .

(i) If $A$ is $C$-bounded, then $A$ is $(C+D)$-bounded. Furthermore, if the relative bound of $A$ with respect to $C$ is 0 , then the relative bound of $A$ with respect to $C+D$ is 0 .

(ii) If $A$ is $C$-compact, then $A$ is $(C+D)$-compact.

PROOF. For (i), we have $D(C) \subseteq D(D), \quad D(C) \subseteq D(A), \quad\|D y\| \leq K_{1}\|y\|+\varepsilon\|C y\|$ $\left(y \in D(C)\right.$ ) for some $K_{1}>0$ and $\varepsilon \in(0,1)$, and $\|A y\| \leq K_{2}\|y\|+\delta\|C y\| \quad(y \in D(C)$ ) for some $K_{2}, \delta>0$. Therefore, $D(C+D)=D(C) \subseteq D(A)$. Fix $y \in D(C)$. Then

$$
\begin{gathered}
\|A y\| \leq K_{2}\|y\|+\delta\|(C+D) y-D y\| \leq K_{2}\|y\|+\delta\|(C+D) y\|+\delta\|D y\| \\
\leq\left(K_{2}+\delta K_{1}\right)\|y\|+\delta\|(C+D) y\|+\delta \varepsilon\|C y\| .
\end{gathered}
$$


Noting that $\quad\|C y\| \leq\|(C+D) y\|+\|D y\| \leq\|(C+D) y\|+K_{1}\|y\|+\varepsilon\|C y\|, \quad$ we obtain $\|C y\| \leq\left(\frac{1}{1-\varepsilon}\right)\|(C+D) y\|+\left(\frac{K_{1}}{1-\varepsilon}\right)\|y\|$. Hence $\|A y\| \leq K_{3}\|y\|+\left(\frac{\delta}{1-\varepsilon}\right)\|(C+D) y\|$, where $K_{3}$ is independent of $y$. Therefore, $A$ is $(C+D)$-bounded and the statement concerning relative bounds follows easily.

For (ii), suppose $\left\{y_{n}\right\}$ is $(C+D)$-bounded, i.e., $y_{n} \in D(C+D)$ and $\left\|y_{n}\right\|+\left\|(C+D) y_{n}\right\| \leq K \quad$ for some constant $K$ independent of $n$. Then $y_{n} \in D(C)$ and $\left\|C y_{n}\right\| \leq\left\|(C+D) y_{n}\right\|+\left\|D y_{n}\right\| \leq K+K_{1}\left\|y_{n}\right\|+\varepsilon\left\|C y_{n}\right\|$ by the $C$-boundedness of $D$. Since $0<\varepsilon<1$ and $\left\|y_{n}\right\| \leq K$, we have $\left\|C y_{n}\right\| \leq \frac{K\left(1+K_{1}\right)}{1-\varepsilon}$. Therefore, $\left\{y_{n}\right\}$ is $C$-bounded. Since $A$ is $C$-compact, $\left\{A y_{n}\right\}$ contains a convergent subsequence. Since $\left\{y_{n}\right\}$ was an arbitrary $(C+$ $D$ )-bounded sequence, $A$ is $(C+D)$-compact.

LEMMA 1.2. Let $B, L$, and $T$ be the operators in Theorems 1.1 and 1.2. Then:

(i) $B$ is $L$-bounded if and only if $B$ is $T$-bounded. Further, the relative bound for $B$ with respect to $L$ is $O$ if and only if the relative bound for $B$ with respect to $T$ is 0 .

(ii) $B$ is L-compact if and only if $B$ is $T$-compact.

PROOF. Consider the differential expression $\left(\frac{1}{a_{n}}\right) l-\tau=\frac{1}{W^{1 / p}} \sum_{i=0}^{n-1}\left(\frac{a_{i}}{a_{n}}\right) P_{i}^{1 / p} D^{i}$. Its coefficients satisfy the perturbation conditions (1.1) since for $t \in I$ and $0 \leq i \leq n-1$,

$$
\frac{1}{s(t)} \int_{1}^{t+\delta s(t)}\left|\frac{\mathrm{a}_{1}}{\mathrm{a}_{\mathrm{n}}}\right|^{p} \frac{p_{1}}{w s^{(\alpha+1) p}}=\frac{1}{s(t)} \int_{t}^{t+\delta s(t)}\left|\frac{\mathrm{a}_{1}}{\mid \mathrm{a}_{\mathrm{n}}}\right|^{p} \leq \text { (constant) } \cdot \delta
$$

by the hypotheses that $\frac{1}{\mathrm{a}_{\mathrm{n}}}, \mathrm{a}_{1}(0 \leq i \leq n-1) \in L^{-}(I)$. Hence by Theorem 1.1(i), $\left(\frac{1}{a_{n}}\right) \mathrm{L}-\mathrm{T}$ is $T$-bounded with relative bound 0. Application of Lemma 1.1 (with $\mathrm{A}=\mathrm{D}=\left(\frac{1}{a_{n}}\right) \mathrm{L}-\mathrm{T}$ and $\mathrm{C}=\mathrm{T}$ ) yields that $\left(\frac{1}{a_{n}}\right) \mathrm{L}-\mathrm{T}$ is $\left\{\mathrm{T}+\left[\left(\frac{1}{a_{n}}\right) \mathrm{L}-\mathrm{T}\right]\right\}=\left(\frac{1}{a_{n}}\right) \mathrm{L}$-bounded with relative bound 0 .

(i) Suppose $B$ is $L$-bounded. Then $B$ is $\left(\frac{1}{a_{n}}\right) \mathrm{L}$-bounded since $\frac{1}{a_{n}} \in L^{-\mu}(I)$. Another application of Lemma 1.1 (with $A=B, C=\frac{1}{a_{n}} L$, and $D=\mathrm{T}-\frac{1}{a_{n}} \mathrm{~L}$ ) shows that $B$ is $T$ bounded.

Next, suppose $B$ is $T$-bounded. By Lemma 1.1 (with $A=B, C=T$, and $D=$ $\left.\left(\frac{1}{a_{n}}\right) \mathrm{L}-\mathrm{T}\right), \quad B$ is $\left(\frac{1}{a_{n}}\right) \mathrm{L}$-bounded. Hence $B$ is $L$-bounded. The statement about zero relative bounds also follows from Lemma 1.1.

(ii) This part is proved in a similar manner using Lemma 1.1(ii). 
(i) Sufficiency. Suppose (1.42) holds for $0 \leq j \leq n-1$ and some $\delta \in\left(0,1 /\left(2 N_{0}\right)\right)$. By Theorem 1.1(i), $B$ is $T$-bounded with relative bound 0 . Hence Lemma 1.3 implies that $B$ is $L$ bounded with relative bound 0 . The result $D\left(L_{l+v}\right)=D(L)$ follows by the same argument used in showing that $D\left(T_{\tau+v}\right)=D(T)$ in the proof of Theorem 1.1.

Necessity. Suppose $B$ is $L$-bounded. Then $B$ is $T$-bounded by Lemma 1.2. Hence by Theorem 1.1, $b,(0 \leq j \leq n-1)$ satisfy (1.42) for some $\delta \in\left(0,1 /\left(2 N_{0}\right)\right)$.

(ii) Sufficiency. Suppose (1.43) holds for $0 \leq j \leq n-1$ and some $\delta \in\left(0, \frac{1}{2 N_{0}}\right)$. Then by Theorem 1.1, $B$ is $T$-compact and hence $L$-compact by Lemma 1.2. The invariance of the essential spectrum and Fredholm index of $L$ under perturbations by $B$ follow as in the proof of Theorem 1.1.

Necessity. Suppose $B$ is $L$-compact. Then $B$ is $T$-compact by Lemma 1.2. By Theorem 1.1, there exists $\delta \in\left(0,1 /\left(2 N_{0}\right)\right)$ such that $b,(0 \leq j \leq n-1)$ satisfy (1.43).

REMARK. Theorems 1.1 and 1.2 apply to operators $T$ and $L$ with coefficients eventually bounded above by the corresponding coefficients of an Euler operator. To see this, note that the hypothesis $\left|s^{\prime}(t)\right| \leq N_{0}$ a.e. on $I$ implies that there exists a positive constant $C$ such that $s(t) \leq C t$ for all $t$ sufficiently large. Now, by definition of $P_{t}$ and $W$ and the hypothesis that $a_{t} \quad(0 \leq i \leq n)$ $\in L^{\infty}(I)$, we have

$$
\frac{\left|a_{t}(t)\right| P_{t}(t)^{1 / p}}{\mathrm{~W}(\mathrm{t})^{1 / p}}=\left|a_{t}(t)\right| s(t)^{t} \leq C_{t} t^{t}
$$

for all $t$ sufficiently large, where $C_{1}$ are constants independent of $t$ and $0 \leq i \leq n$.

EXAMPLE 1.1. Let $n=2, p=2, w \equiv 1, \alpha=0$, and $s$ be any positive, $\operatorname{AC}_{\mathrm{loc}}([a, \infty))$ function such that $\left|s^{\prime}(t)\right| \leq N_{0}$ for $t \in I=[a, \infty)$. Then $W \equiv 1$ and $P_{1}(t)=s(t)^{2 t}$ for $i=0,1,2$. Consider

$$
L y=a_{2}(t) s(t)^{2} y^{\prime \prime}+a_{1}(t) s(t) y^{\prime}+a_{0}(t) y
$$

and

$$
B y=b_{1}(t) y^{\prime}+b_{0}(t) y,
$$

where $\frac{1}{a_{2}}, a_{0}, a_{1}, a_{2} \in L^{\infty}(I)$ and $b_{0}, b_{1} \in L_{\text {oc }}^{2}(I)$. Then

$$
g_{, . \delta}(t)=\frac{1}{s(t)} \int_{1}^{t+\delta s(t)} \frac{\left|b_{J}(\tau)\right|^{2}}{s(\tau)^{2 j}} d \tau \quad(j=0,1)
$$

By Theorem 1.2, $B$ is $L$-bounded if and only if $\sup _{t \in l} g_{ر, \delta}(t)<\infty \quad(j=0,1)$ and $L$-compact if and only if $\lim _{t \rightarrow \infty} g_{\text {J. }}(t)=0 \quad(j=0,1)$ for some $\delta \in\left(0,1 /\left(2 N_{0}\right)\right)$. 
Next we prove a corollary of Theorem 1.2 in which an $n$th order perturbation $B$ of $L$ is considered. The perturbation is such that the coefficients of the highest-order terms in $L$ and $L+B$ obey the same hypotheses. Before stating the corollary, we prove a lemma concerning the domains of the single-term operator $T$ and multi-term operator $L$.

LEMMA 1.3. Let $T$ and $L$ be as in Theorems 1.1 and 1.2. Then $D(L)=D(T)$.

PROOF. First consider the case in which $a_{n} \equiv 1$. By Theorem 1.1 with $v=\frac{1}{W^{1 / p}} \sum_{i=0}^{n-1} a_{i} P_{t}^{1 / p} D^{i}, B$ is $T$-bounded and $L=T_{\tau+v}=T+B$. Thus $D(T) \subseteq D(B)$, and so $D(L)=D(T+B)=D(T)$. For general $a_{n}$ such that $a_{n}, 1 / a_{n} \in L^{-}(I)$, we may replace $T$ by $a_{n} T$ without affecting $T$-boundedness of $B$. It follows that $D(L)=D\left(a_{n} T\right)=D(T)$.

COROLLARY 1.1. Let $p, s, w, W, P_{1}$, and $L$ be as in Theorem 1.2. Let $B: L_{w}^{p}(a, \infty) \rightarrow L_{w}^{p}(a, \infty)$ be the maximal operator corresponding to

$$
v=\frac{1}{W^{1 / p}}\left\{b_{n} P_{n}^{1 / p} D^{n}+\sum_{j=0}^{n-1} b_{j} D^{\prime}\right\}
$$

where $b_{n}, \frac{1}{a_{n}+b_{n}} \in L^{-\infty}(I), \quad b, \in L_{l o c}^{p}(I) \quad(0 \leq j \leq n)$,

$$
\lim _{t \rightarrow \infty} \frac{1}{s(t)} \int_{t}^{t+\delta s(t)}\left|b_{n}(\tau)\right|^{p} d \tau=0
$$

and

$$
\lim _{t \rightarrow \infty} \frac{1}{s(t)} \int_{t}^{t+\delta s(t)} \frac{\left|b_{j}(\tau)\right|^{p}}{w(\tau) s(\tau)^{(\alpha+j) p}} d \tau=0 \quad(0 \leq j \leq n-1)
$$

for some $\delta \in\left(0,1 /\left(2 N_{0}\right)\right)$. Let $R: L_{w}^{p}(a, \infty) \rightarrow L_{w}^{p}(a, \infty)$ be the maximal operator corresponding to $l+v$. Then $\quad D(L)=D(R), \quad \sigma_{e}(L)=\sigma_{e}(R), \quad$ and $\lambda \in \rho_{e}(L) \Rightarrow \kappa(\lambda I-L)=\kappa(\lambda I-R)$.

PROOF. In view of Theorem 1.2, it suffices to prove the corollary for the operator $R=L+\frac{1}{W^{1 / p}} b_{n} P_{n}^{1 / p} D^{n} . \quad$ As in Theorem 1.1, let $T: L_{w}^{p}(a, \infty) \rightarrow L_{w}^{p}(a, \infty)$ be the maximal operator corresponding to $\tau=\frac{1}{W^{1 / p}} P_{n}^{1 / p} D^{n}$. Then $R=L+b_{n} T$. By Lemma 1.3, $D(L)=D(T)$ and $D(R)=D(T)$. Hence $D(L)=D(R)$. For any scalar $\lambda$ and $y \in D(R)=D(L)$,

$$
\begin{aligned}
& (\lambda I-R) y=\lambda y-L y-\frac{1}{W^{1 / p}} b_{n} P_{n}^{1 / p} y^{(n)} \\
& \quad=\lambda y-L y+\frac{b_{n}}{a_{n}}\left\{\lambda y-L y+\frac{1}{W^{1 / p}} \sum_{k=0}^{n-1} a_{k} P_{k}^{1 / p} y^{(k)}-\lambda y\right\}=A_{\lambda} y+S_{\lambda} y
\end{aligned}
$$


where $A_{\lambda}$ and $S_{\lambda}$ are the maximal operators associated with $\left(1+\frac{b_{n}}{a_{n}}\right)(\lambda I-l)$ and $\frac{1}{W^{1 / p}} \sum_{k=0}^{n-1} b_{n} \frac{a_{k}}{a_{n}} P_{k}^{1 / p} D^{k}-b_{n} \frac{\lambda}{a_{n}} I$, respectively. An application of Theorem 1.2 (with $L, B$, and $L_{l+v}$ replaced by $A_{\lambda}, S_{\lambda}$, and $\lambda I-R$, respectively) yields that $S_{\lambda}$ is $A_{\lambda}$-compact, $\sigma_{e}\left(A_{\lambda}\right)=\sigma_{e}(\lambda I-R)$, and

$$
0 \in \rho_{e}\left(A_{\lambda}\right) \Rightarrow \kappa\left(A_{\lambda}\right)=\kappa(\lambda I-R)
$$

By definition of $A_{\lambda}, \quad \lambda I-L=\left(\frac{a_{n}}{a_{n}+b_{n}}\right) A_{\lambda} . \quad$ Let $h=\frac{a_{n}}{a_{n}+b_{n}} . \quad$ Then $h$, $1 / h \in L^{\infty}(I)$ and $R(\lambda I-L)=\left\{h g: g \in R\left(A_{\lambda}\right)\right\}$. The result that $R\left(A_{\lambda}\right)$ is closed if and only if $R(\lambda I-L)$ is closed follows from the next lemma.

LEMMA 1.4. Let $M$ be a closed subspace of $L_{w}^{p}(a, \infty)$ and $N=h M=\{h g: g \in M\}$, where $h, 1 / h \in L^{\infty}(a, \infty)$. Then $N$ is closed.

PROOF. Suppose $h g_{n} \in N$ with $g_{n} \in M$ and $h g_{n} \rightarrow z$. Since $1 / h \in L^{-\infty}(a, \infty)$, $\dot{g}_{n} \rightarrow z / h$. Since $M$ is closed, $z / h \in M$. Therefore, $z=h \cdot(z / h) \in N$. So $N$ is closed.

Since $\sigma_{e}\left(A_{\lambda}\right)=\sigma_{e}(\lambda I-R), \quad \rho_{e}\left(A_{\lambda}\right)=\rho_{e}(\lambda I-R)$, i.e.,

$$
\left\{\mu: R\left(\mu I-A_{\lambda}\right) \text { is closed }\right\}=\{\mu: R(\mu I-(\lambda I-R)) \text { is closed }\} .
$$

Therefore, $R\left(A_{\lambda}\right)$ closed $\quad \Leftrightarrow \quad R(\lambda I-R)$ closed. It follows that $\rho_{e}(L)=\rho_{e}(R)$; and so $\sigma_{e}(L)=\sigma_{e}(R)$.

It remains to show that $\lambda \in \rho_{e}(L) \Rightarrow \kappa(\lambda I-L)=\kappa(\lambda I-R)$. Let $\lambda \in \rho_{e}(L)$. Then $R(\lambda I-L)$ is closed and $L_{w}^{p}(a, \infty)=R(\lambda I-L) \oplus M$, where $M=N\left(\bar{\lambda} I-L^{*}\right)$. Since $L^{*} y=\bar{\lambda} y$ has at most $n L_{w}^{p}(a, \infty)$ solutions, $M$ is finite-dimensional.

Let $\psi=\frac{a_{n}+b_{n}}{a_{n}}$. Then $\psi, \frac{1}{\psi} \in L^{\infty}(I)$ and $A_{\lambda}=\psi(\lambda I-L)$. Any $f \in L_{w}^{p}(a, \infty)$ can be written as $f=(\lambda I-L) g+m$, where $g \in D(L)$ and $m \in M$. Thus $\psi f=\psi(\lambda I-L) g+\psi m$ with $\psi f \in L_{w}^{p}(a, \infty), \psi(\lambda I-L) g \in R\left(A_{\lambda}\right)$, and $\psi m \in \psi M$. Now, since $R(\lambda I-L)$ closed $\Rightarrow$ $R\left(A_{\lambda}\right)$ closed, $L_{w}^{p}(a, \infty)=R\left(A_{\lambda}\right) \oplus N$ where $N=\psi M=\{\psi m: m \in M\}$. Since $\psi$, $\frac{1}{\psi} \in L^{\infty}(a, \infty), \operatorname{dim} N=\operatorname{dim} M$. By definition, the deficiency index of $A_{\lambda}$ is

$$
\begin{aligned}
\beta\left(A_{\lambda}\right) & =\operatorname{dim}\left[L_{w}^{p}(a, \infty) \backslash R\left(A_{\lambda}\right)\right]=\operatorname{dim} N=\operatorname{dim} M \\
& =\operatorname{dim}\left[L_{w}^{p}(a, \infty) \backslash R(\lambda I-L)\right]=\beta(\lambda I-L)
\end{aligned}
$$

Since $A_{\lambda}=\psi(\lambda I-L)$ and $\psi \neq 0$ (because $\left.\frac{1}{\psi} \in L^{\infty}(a, \infty)\right), N\left(A_{\lambda}\right)=N(\lambda I-L)$. Therefore, $\alpha\left(A_{\lambda}\right)=\alpha(\lambda I-L)$. Thus $\kappa\left(A_{\lambda}\right)=\kappa(\lambda I-L)$. Since $R\left(A_{\lambda}\right)$ is closed, $0 \in \rho_{e}\left(A_{\lambda}\right)$. Hence by (1.50), $\kappa\left(A_{\lambda}\right)=\kappa(\lambda I-R)$. Therefore, $\kappa(\lambda I-L)=\kappa(\lambda I-R)$. 
REMARK. Note that (1.49) and (1.43) are identical conditions on the lower-order perturbation coefficients $b_{j}, \quad 0 \leq j \leq n-1$. Theorem 1.2 is a result for lower-order perturbations of $L=\frac{1}{W^{1 / p}} \sum_{i=0}^{n} a_{1} P_{1}^{1 / p} D^{\prime}$, where $\frac{1}{a_{n}}, a_{1}(0 \leq i \leq n-1) \in L^{\infty}(a, \infty)$. Corollary 1.1 applies to $n$th order perturbations of $L$ of the form $R=\frac{1}{W^{1 / p}}\left\{\left(a_{n}+b_{n}\right) P_{n}^{1 / p} D^{n}+\sum_{i=0}^{n-1}\left(a_{1} P_{1}^{1 / p}+b_{1}\right) D^{i}\right\}$, where $b_{n}$ satisfies (1.48) and $a_{n}+b_{n}, \frac{1}{a_{n}+b_{n}} \in L^{\infty}(a, \infty)$ (in analogy to the conditions on $a_{n}$ in the operator $L$ ).

\section{CONDITIONS FOR OPERATORS WITH LARGE COEFFICIENTS}

Recall that Theorem 1.1 applies to operators

$$
T=\frac{1}{W^{1 / p}} P^{1 / p} D^{n}
$$

such that

$$
\left[\frac{\mathrm{P}(\mathrm{t})}{\mathrm{W}(\mathrm{t})}\right]^{1 / p} \leq C t^{n}
$$

for some constant $C$ and all $t$ sufficiently large. The following theorem generalizes the sufficiency conditions in Theorem 1.1 for operators $T$ with arbitrarily large coefficients.

THEOREM 2.1. Let $1<p<\infty$ and $I=[a, \infty)$. Let $P$ and $W$ be nondecreasing, positive continuous functions on I such that $W^{-q / p}, P^{-q / p} \in L_{l i c}(I)$, where $\frac{1}{p}+\frac{1}{q}=1$. Let $T$, $B: L_{w}^{p}(I) \rightarrow L_{w}^{p}(I)$ be the maximal operators corresponding to

$$
\tau=\frac{1}{W^{1 / p}} P^{1 / p} D^{n}
$$

and

$$
v=\frac{1}{W^{1 / p}} \sum_{j=0}^{n-1} b, D^{j}
$$

respectively, where each $b, L_{l o c}^{p}(I)$. For $0 \leq j \leq n-1$ and $\delta>0$, define

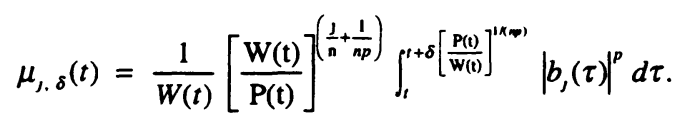

(i) If there exists $\delta>0$ such that

$$
\sup _{t \in I} \mu_{J . \delta}(t)<\infty \quad(0 \leq j \leq n-1)
$$




$$
\lim _{t \rightarrow \infty} \mu_{j, \delta}(t)=0 \quad(0 \leq j \leq n-1)
$$

then $B$ is T-compact.

PROOF. (i) Suppose (2.1) holds for some $\delta>0$. We will show that Theorem $A$ applies to the choices $f=\left(\frac{P}{W}\right)^{1 /(n p)}, N=|b|^{p}$, and $\varepsilon_{0}=\delta$. Fix $t \in I$ and $\varepsilon \in(0, \delta)$. Since $P$ is nondecreasing on $I$, it follows that

$$
T_{t, \varepsilon}(P)=\left\{\frac{1}{\varepsilon f(t)} \int_{t}^{t+\varepsilon f(t)} \frac{1}{P(\tau)^{q / p}} d \tau\right\}^{p / q} \leq \frac{1}{P(t)}
$$

Similarly, $T_{t, \varepsilon}(W) \leq \frac{1}{W(t)}$. The choice $f=\left(\frac{P}{W}\right)^{1 /(n p)}$ is made so that certain upper bounds on $S_{1}(\varepsilon)$ and $S_{2}(\varepsilon)$ are equal: $\quad S_{k}(\varepsilon) \leq \frac{1}{\varepsilon} \sup _{t \in I} \mu_{,, \delta}(t) \quad(k=1,2)$. By $(2.1)$, there exists a constant $C$ independent of $\varepsilon$ such that $S_{k}(\varepsilon) \leq \frac{C}{\varepsilon}$ for $k=1,2$ and $\varepsilon \in(0, \delta)$. Hence by Theorem A, there is a constant $K$ such that

$$
\int_{I}\left|b, y^{(\jmath)}\right|^{p} \leq K\left\{\frac{1}{\varepsilon^{p p+1}} \int_{I} W|y|^{p}+\varepsilon^{(n-\jmath) p-1} \int_{I} P\left|y^{(n)}\right|^{p}\right\}
$$

for all $y \in D(T)$. By the same calculations used to obtain (1.8) in the proof of Theorem 1.1, $\|B y\| \leq K_{1} \varepsilon^{(-n+1-1 / p)}\|y\|+K_{1} \varepsilon^{(1-1 / p)}\|T y\|, \quad K_{1}=K^{1 / p}$, for all $y \in D(T)$. Since $p>1$, the coefficient of $\|T y\|$ can be made arbitrarily small by choosing $\varepsilon \in(0, \delta)$ sufficiently small. Therefore, $B$ is $T$-bounded with relative bound 0 .

(ii) Suppose (2.2) holds for some $\delta>0 . T$-compactness of $B$ follows by the argument used in proving sufficiency in Theorem 1.1(ii).

EXAMPLE 2.1. Let $W(t) \equiv 1$ and $P(t)=e^{t}$. Then $T=e^{t / p} D^{n}$ and $B=\sum_{j=0}^{n-1} b, D^{j}$. In this case, condition (2.1) precludes exponential growth of $b$. Suppose

$$
\left|b_{j}(t)\right| \leq C, t^{\Delta,}, \quad a \leq t<\infty, \quad 0 \leq j \leq n-1, \quad \Delta_{j} \geq 0
$$

for some constants $C_{j}$ and $\Delta_{j}$. Fix $j$ and let $\Delta=\Delta$, and $C=C_{j}^{p}$. Then by the definition of $\mu_{J, \delta}$ in Theorem 2.1,

$$
\begin{aligned}
\mu_{, \delta}(t) & \leq \frac{C}{e^{(j / n+1 /(n p)) t}} \int_{t}^{t+\delta e^{i(\Delta p)}} \tau^{\Delta p} d \tau \\
& =\frac{C}{(\Delta p+1) e^{(j / n+1 /(n p)) t}}\left[\left(t+\delta e^{t /(n p)}\right)^{\Delta p+1}-t^{\Delta p+1}\right] .
\end{aligned}
$$

For $t$ sufficiently large, we obtain (with a different constant)

$$
\mu_{J, \delta}(t) \leq \frac{C}{e^{(J / n+1 /(n p)) t}} e^{(\Delta p+1) t /(n p)}=C e^{(\Delta-\jmath) t / n}
$$


Hence (2.1) holds if $\Delta \leq j$, and (2.2) holds if $\Delta<j$. For example, the Euler operator $\sum_{j=0}^{n-1} t^{\prime} D^{\prime}$ is $T$-bounded, and the operator $\sum_{j=0}^{n-1} t^{t^{-\varepsilon}} D^{\prime} \quad(\varepsilon>0)$ is $T$-compact.

We state here another part of Theorem 2.1 from Brown and Hinton [3] mentioned earlier.

THEOREM B. Let $1 \leq p<\infty, I=[a, \infty)$, and $0 \leq j \leq n-1$. Let $N, W$, and $P$ be positive measurable functions such that $N \in L_{l o x}(I)$; for $p>1, W^{-q / p}, P^{-q / p} \in L_{l o c}(I)$ where $\frac{1}{p}+\frac{1}{q}=1 ;$ for $p=1, W^{-1}, P^{-1}$ are locally essentially bounded on I. Define

$$
T_{t, \varepsilon}(P)=\left\{\begin{array}{cl}
\left\|P^{-1}\right\|_{\infty,|t, t+\varepsilon f|}, & p=1 \\
{\left[\frac{1}{\varepsilon f} \int_{t}^{t+\varepsilon f} P^{-q / p}\right]^{p / q},} & 1<p<\infty
\end{array}\right.
$$

with similar definitions for $T_{t, \varepsilon}(W)$. Suppose there exists $\varepsilon_{0}>0$ and a positive continuous function $f=f(t)$ on I such that $f^{\prime}(t) \geq 0$,

$$
R_{1}(\varepsilon):=\sup _{t \in I}\left\{f(t)^{(n-\jmath) p} N(t) T_{t, \varepsilon}(P)\right\}<\infty
$$

and

$$
R_{2}(\varepsilon):=\sup _{t \in I}\left\{f(t)^{-J p} N(t) T_{t, \varepsilon}(W)\right\}<\infty
$$

for all $\varepsilon \in\left(0, \varepsilon_{0}\right)$. Then there exists $K>0$ such that for all $\varepsilon \in\left(0, \varepsilon_{0}\right)$ and $y \in D$,

$$
\int_{1} N\left|y^{(\jmath)}\right|^{p} \leq K\left\{\varepsilon^{-\jmath p} R_{2}(\varepsilon) \int_{1} W|y|^{p}+\varepsilon^{(n-j) p} R_{1}(\varepsilon) \int_{1} P\left|y^{(n)}\right|^{p}\right\}
$$

where $D=\left\{y: y^{(n-1)} \in A C_{l o c}(I), \int_{l} W|y|^{p}<\infty\right.$, and $\left.\int_{I} P\left|y^{(n)}\right|^{p}<\infty\right\}$.

This result can be used to prove the following theorem, which gives pointwise conditions sufficient for relative boundedness and relative compactness.

THEOREM 2.2. Suppose the conditions in Theorem 2.1 are satisfied with the definition of $\mu_{J, \delta}$ replaced by

$$
\mu_{j}(t)=\frac{1}{W(t)}\left[\frac{W(t)}{P(t)}\right]^{\prime n}\left|b_{j}(t)\right|^{p} \quad(0 \leq j \leq n-1)
$$

In addition, suppose $\frac{P}{W} \in A C_{l a c}(I)$ with $\frac{d}{d t}\left[\frac{P(t)}{W(t)}\right] \geq 0$ for $t \in I$. Then the conclusions in Theorem 2.1 hold for $1 \leq p<\infty$ provided that for the case $p=1, W^{-1}$ and $P^{-1}$ are locally essentially bounded on $I$.

PROOF. (i) Suppose $\sup _{t \in t} \mu_{t}(t)<\infty$ for $0 \leq j \leq n-1$. We will show that Theorem B applies to the choices $f=\left(\frac{P}{W}\right)^{1 /(n p)}, N=|b,|^{p}$, and any $\varepsilon_{0}>0$. Fix $t \in I$ and $\varepsilon>0$. Since 
$P$ and $W$ are nondecreasing on $I, \quad T_{t, \varepsilon}(P) \leq \frac{1}{P(t)} \quad$ and $\quad T_{t, \varepsilon}(W) \leq \frac{1}{W(t)}$. Hence $R_{1}(\varepsilon) \leq \sup _{t \in I}\left\{f(t)^{(n-\jmath) p}\left|b_{\jmath}(t)\right|^{p} \frac{1}{P(t)}\right\}$ and $R_{2}(\varepsilon) \leq \sup _{t \in I}\left\{f(t)^{-\jmath p}\left|b_{J}(t)\right|^{p} \frac{1}{W(t)}\right\}$. By the choice of $f, R_{k}(\varepsilon) \leq \sup _{t \in I} \mu_{j}(t)<\infty \quad(k=1,2)$. Therefore, Theorem B applies. The rest of the proof, including (ii), follows as in the proof of Theorem 2.1.

EXAMPLE 2.2. Let $W(t) \equiv 1$ and $P(t)=e^{\alpha t}, \alpha>0$. Then $T=e^{\alpha / 1 p} D^{n}$ and $B=\sum_{j=0}^{n-1} b_{j} D^{\prime}$. Let $1 \leq p<\infty$. Suppose $\left|b_{J}(t)\right| \leq C_{j} e^{\beta, \prime}, a \leq t<\infty, 0 \leq j \leq n-1$, for some constants $C_{J}$ and $\beta_{j}$. Then $\mu_{j}(t)=\frac{1}{e^{\alpha_{J} / / n}}\left|b_{J}(t)\right|^{p} \leq C_{J}^{p} e^{\left(\beta_{j} p-\alpha / n\right) t}$. Thus by Theorem 2.2, $\beta, \leq \frac{\alpha j}{n p} \Rightarrow B$ is $T$-bounded and $\beta,<\frac{\alpha j}{n p} \Rightarrow B$ is $T$-compact.

So the pointwise conditions on $b_{j}$ in Theorem 2.2 allow $b_{j}$ to grow exponentially. In contrast, the integral average conditions of Theorem 2.1 applied to Example 2.1 allow polynomial, but not exponential, growth of $b$,

\section{INTEGRAL AVERAGE CONDITIONS FOR THE CASE $p=1$}

The following theorem gives sufficient conditions for $T$-boundedness for the case $p=1$ for integral averages.

THEOREM 3.1. Let $P$ and $W$ be nondecreasing, positive continuous functions such that $\frac{1}{P}$ and $\frac{1}{W}$ are locally essentially bounded on $[a, \infty)$. Let $T, B: L_{w}^{1}(a, \infty) \rightarrow L_{w}^{1}(a, \infty)$ be the maximal operators corresponding to

and

$$
\tau=\frac{1}{W} P D^{n}
$$

$$
v=\frac{1}{W} \sum_{\mathrm{j}=0}^{\mathrm{n}-1} b, D^{\prime},
$$

respectively, where each $b$, is a measurable function on $[a, \infty)$. For $0 \leq j \leq n-1$ and $\delta>0$, define

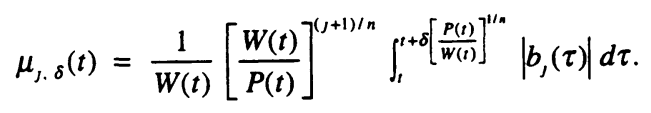

If there exists $\delta>0$ such that

$$
\sup _{a \leq t<\infty} \mu_{, \delta}(t)<\infty \quad(0 \leq j \leq n-1)
$$

then $B$ is $T$-bounded. If in addition $b_{n-1} \equiv 0$, then the relative bound of $B$ with respect to $T$ is 0 . PROOF. We show that Theorem A applies to the choices $f=\left(\frac{P}{W}\right)^{1 / n}, p=1, N=\left|b_{\jmath}\right|$, and any $\varepsilon_{0}=\delta$. Fix $t \in[a, \infty)$ and $\varepsilon \in(0, \delta)$. Using the hypothesis that $P$ is nondecreasing, we have $T_{t, \varepsilon}(P)=\left\|\frac{1}{P}\right\|_{\infty,|t, t+\varepsilon f(t)|} \leq \frac{1}{P(t)}$. Similarly, $T_{t, \varepsilon}(W) \leq \frac{1}{W(t)}$. These inequalities yield upper bounds for $S_{1}(\varepsilon)$ and $S_{2}(\varepsilon)$. The choice $f=\left(\frac{P}{W}\right)^{1 / n}$ is made so that these upper bounds are equal: for $k=1$ or $2, S_{k}(\varepsilon) \leq \frac{1}{\varepsilon} \sup _{a \leq t<\infty} \mu_{, \delta}(t) \leq \frac{M}{\varepsilon}$, where the last inequality 
follows by hypothesis (for some constant $M>0$ ). By Theorem A, there exists $K>0$ such that for all $\varepsilon \in(0, \delta)$ and $y \in D(T)$,

$$
\int_{a}^{\infty}\left|b, y^{(\jmath)}\right| \leq K\left\{\varepsilon^{-\jmath} S_{2}(\varepsilon) \int_{a}^{\infty} W|y|+\varepsilon^{n-\jmath} S_{1}(\varepsilon) \int_{a}^{\infty} P\left|y^{(n)}\right|\right\}
$$

Let $\|\bullet\|$ denote the norm of $L_{w}^{1}(a, \infty)$. Then

$$
\begin{aligned}
& \|B y\| \leq \sum_{j=0}^{n-1}\left\|\frac{1}{W} b_{j} y^{(j)}\right\|=\sum_{j=0}^{n-1} \int_{a}^{\infty}\left|b, y^{(\jmath)}\right| \\
& \leq K \sum_{j=0}^{n-1}\left\{\varepsilon^{-\jmath} S_{2}(\varepsilon)\|y\|+\varepsilon^{n-\jmath} S_{1}(\varepsilon)\|T y\|\right\} \leq K M \sum_{j=0}^{n-1}\left\{\varepsilon^{-j-1}\|y\|+\varepsilon^{n-j-1}\|T y\|\right\}
\end{aligned}
$$

where we have used the estimates on $S_{1}$ and $S_{2}$. Hence $B$ is $T$-bounded.

If $b_{n-1} \equiv 0$, then the previous sum can be truncated at $j=n-2$ : $\|B y\| \leq C(\varepsilon)\|y\|+K M\left(\sum_{j=0}^{n-2} \varepsilon^{n-j-1}\right)\|T y\|$ for all $y \in D(T)$, where $C(\varepsilon)$ is independent of $y$. Restrict $\varepsilon \in(0, \delta)$ such that $\varepsilon<1$. Then $\|B y\| \leq C(\varepsilon)\|y\|+K M(n-1) \varepsilon\|T y\|$ for all $y \in D(T)$, from which it follows that the relative bound of $B$ with respect to $T$ is 0 .

ACKNOWLEDGEMENTS. Supported in part by the University of Tennessee Knoxville and Oak Ridge National Laboratory Science Alliance Program. The author would like to thank Professor Don B. Hinton for his advice.

\section{REFERENCES}

1 T.G. Anderson. A Theory of Relative Boundedness and Relative Compactness for Ordinary Differential Operators. Ph.D. thesis, University of Tennessee Knoxville (1989).

2 E. Balslev and T.W. Gamelin. The Essential Spectrum of a Class of Ordinary Differential Operators. Pacific J. Math., 14 (1964), 755-776.

3 R.C. Brown and D.B. Hinton. Sufficient Conditions for Weighted Inequalities of Sum Form. J. Math. Anal. Appl., 112 (1985), 563-578.

4 S. Goldberg. Unbounded Linear Operators: Theory and Applications. (New York: Dover, 1985).

5 T. Kato. Perturbation Theory for Linear Operators. (Berlin: Springer-Verlag, 1966).

$6 \quad$ M.K. Kwong and A. Zettl. Weighted Norm Inequalities of Sum Form Involving Derivatives. Proc. Roy. Soc. Edinburgh Ser. A., 88 (1981), 121-134.

7 M.A. Naimark. Linear Differential Operators, II. (New York: Ungar, 1968). 


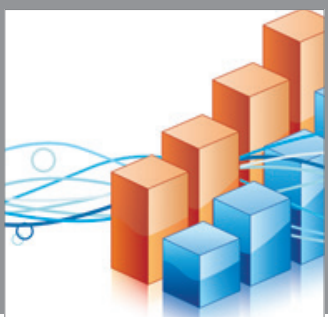

Advances in

Operations Research

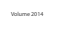

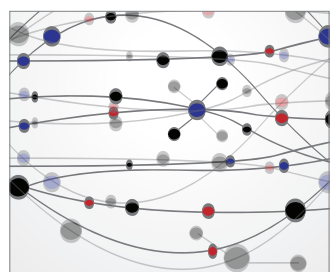

\section{The Scientific} World Journal
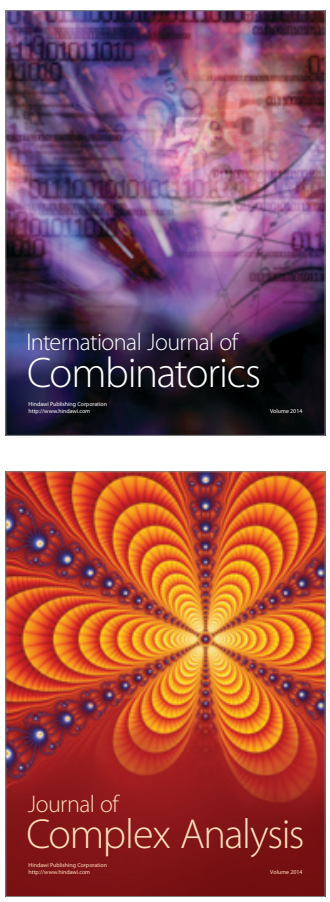

International Journal of

Mathematics and

Mathematical

Sciences
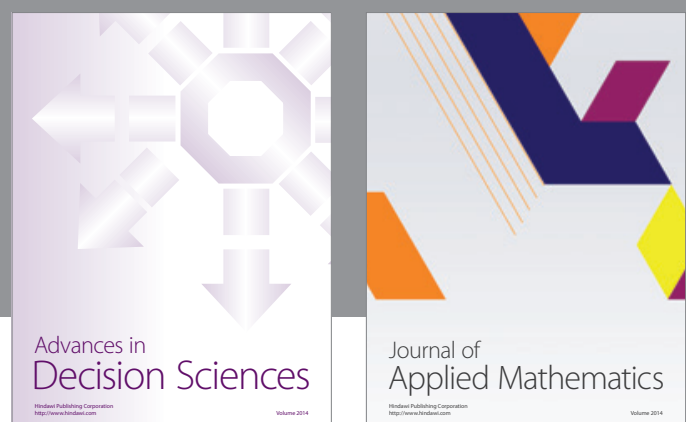

Journal of

Applied Mathematics
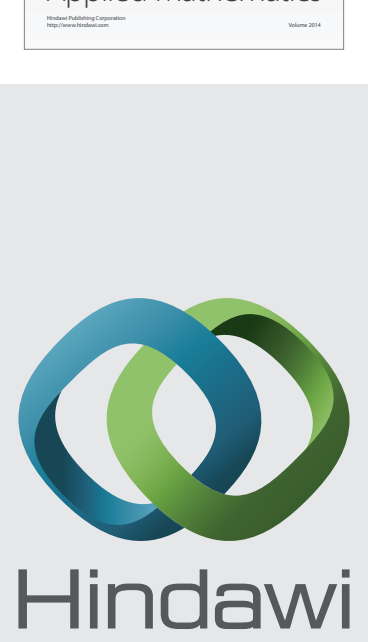

Submit your manuscripts at http://www.hindawi.com


Mathematical Problems in Engineering
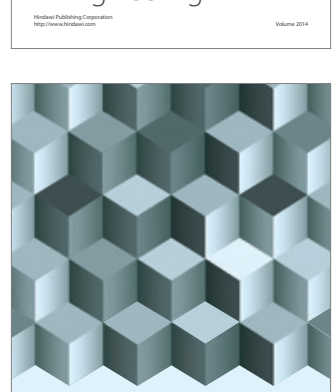

Journal of

Function Spaces
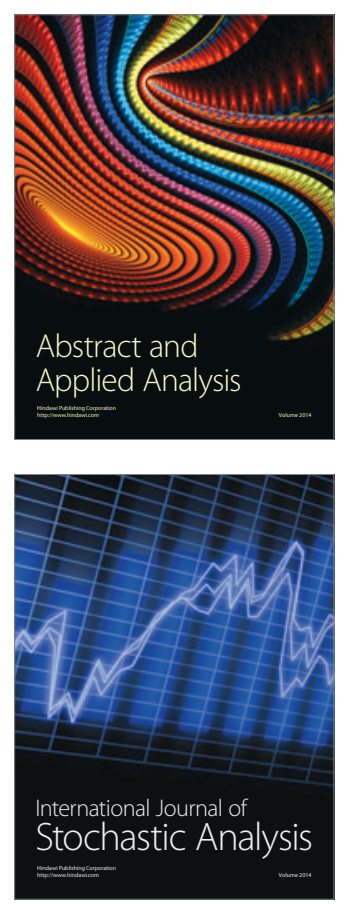

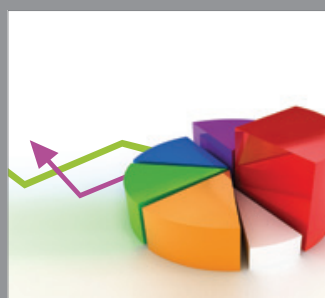

ournal of

Probability and Statistics

Promensencen
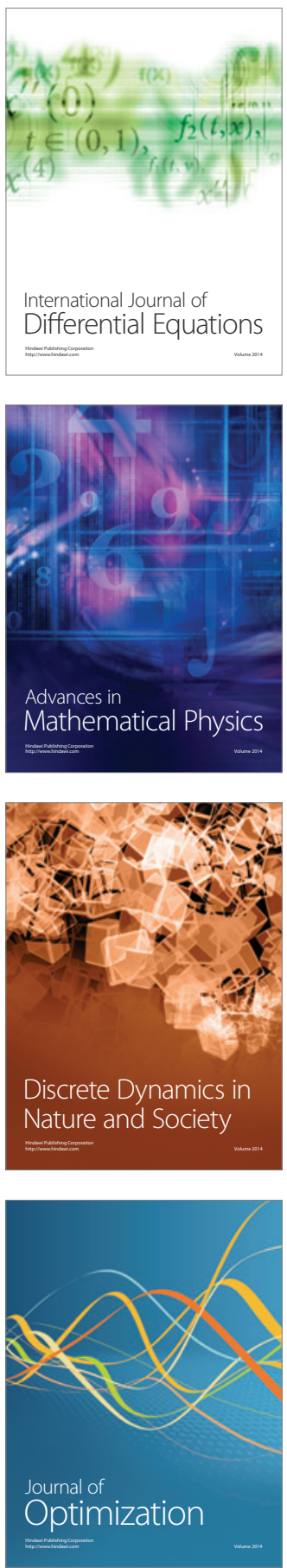